\title{
Registro de Macrodontes paulistus (Gastropoda: Odontostomidae) em um sambaqui na Ilha do Teixeira, baía de Paranaguá, sul do Brasil
}

\author{
Marcos de Vasconcellos Gernet ${ }^{1}$ (D), Carlos Eduardo Belz ${ }^{1}$ (D) Fabiano Pinheiro $^{2} \&$ \\ Carlos João Birckolz ${ }^{1,3}$
}

(1) Universidade Federal do Paraná, Centro de Estudos do Mar, Laboratório de Ecologia Aplicada e Bioinvasões, Avenida Beira Mar, Pontal do Sul 83255-971, Pontal do Paraná, Paraná, Brasil. E-mail: lmv.gernet@gmail.com, belzoceanos@gmail.com

(2) Núcleo Regional de Educação de Paranaguá, Rua Baronesa do Cerro Azul 1027, Campo Grande 83203420, Paranaguá, Paraná, Brasil. E-mail: fabilu51@ hotmail.com

(3) Instituto Chico Mendes de Conservação da Biodiversidade, Parque Nacional de São Joaquim, Avenida Pedro Bernardo Warmling 1542, Esquina 88650-000, Urubici, Santa Catarina, Brasil. E-mail: carlosbirc@gmail.com

Gernet M.V., Belz C.E., Pinheiro F. \& Birckolz C.J. (2020) Registro de Macrodontes paulistus (Gastropoda, Odontostomidae) em um sambaqui na Ilha do Teixeira, baía de Paranaguá, sul do Brasil. Pesquisa e Ensino em Ciências Exatas e da Natureza, 4: e1480. http://dx.doi.org/10.29215/pecen.v4i0.1480

Editor acadêmico: Silvio F. B. Lima. Recebido: 29 junho 2020. Aceito: 15 agosto 2020. Publicado: 17 agosto 2020.

Resumo: Macrodontes paulistus Pilsbry \& Ihering, 1898 é um odontostomídeo com concha fusiforme alongada, carinada e voltas pouco convexas, protoconcha acuminada, sutura profunda e levemente inclinada. Esta espécie foi encontrada em camadas internas de um sambaqui localizado na Ilha do Teixeira, baía de Paranaguá, sul do Brasil. Os exemplares foram coletados manualmente, através de prospecção visual. Três exemplares adultos de $M$. paulistus foram encontrados, todos apresentando conchas com desgastes característicos de material encontrado em sambaquis. Este é o primeiro registro da espécie em sambaquis.

Palavras chave: Litoral do Paraná, molusco, Odontostomidae, sítio arqueológico.

\section{Record of Macrodontes paulistus (Gastropoda, Odontostomidae) in a shell mound on Teixeira Island, Paranaguá bay, southern Brazil}

Abstract: Macrodontes paulistus Pilsbry \& Ihering, 1898 is an odontostomid with fusiform, elongated, carinate shell and slightly convex whorls, acuminated protoconch, deep and slightly inclined suture. This species was found in the inner layers of a shell mound located on Teixeira Island, Paranaguá bay, southern Brazil. The specimens were collected manually, through visual prospecting. Three adult specimens of $\boldsymbol{M}$. paulistus were found, all having shells with characteristic wear of material found in shell mounds. This is the first record of the species in shell mounds.

Key words: Coast of Paraná, mollusk, Odontostomidae, archaeological site.

A família Odontostomidae Pilsbry \& Vanatta, 1898 é um grupo de gastrópodes terrestres que tipicamente apresentam perístoma com dentes e lamelas, alguns bem desenvolvidos e outros simples, cuja finalidade é a proteção contra predadores. Apesar da vantagem, dentes e lamelas podem dificultar a protração e retração do animal na concha (Simone 2018). Odontostomídeos são encontrados na América do Sul (Oliveira \& Almeida 1999; Simone 2006), sendo caracterizados pelas conchas tipicamente fusiformes e de tamanho mediano (cerca de 30 mm de comprimento) (Simone 2006). 
Macrodontes paulistus Pilsbry \& Ihering, 1898 é um odontostomídeo com concha fusiforme alongada, carinada, voltas pouco convexas e coloração castanho-avermelhada. Esta espécie também possui protoconcha tipicamente acuminada, sutura profunda e levemente inclinada, ornamentação delicada composta por estrias irregulares e em zigzag, além de marcas espirais intercaladas perpendicularmente por linhas esparsas; teleoconcha com linhas axiais crenuladas e dispostas sem intervalo; abertura com lamelas internas bem desenvolvidas, um dente menor na região parietal do lábio interno e dois dentes na região palatal, além de um pequeno dentículo na região columelar. O perístoma em conchas de $M$. paulistus é expandido e não há opérculo (Pilsbry 1898; Simone 2006).

Macrodontes paulistus vive associada a áreas de vegetação de Floresta Ombrófila Densa bem preservadas, habitando entre troncos e folhas de plantas arbustivo-arbóreas (Simone 2006; M. V. Gernet, observação pessoal - outubro 2018). Esta espécie está distribuída entre os estados de São Paulo e Paraná (Simone 2006), tendo Iguape (São Paulo) como localidade-tipo (Pilsbry 1898). Diante disso, este trabalho tem como objetivo relatar o registro de $M$. paulistus nas camadas depositadas no sambaqui da Ilha do Teixeira, litoral do Estado do Paraná.

A Ilha do Teixeira (25029'26" S, 48³8'52" O) está localizada na baía de Paranaguá (Figura 1), na área da enseada de Guarapirocaba, apresentando uma extensão territorial de aproximadamente $1.47 \mathrm{~km}^{2}$ (Maack 1969). De acordo com APPA et al. (2018), esta área tem grandes restrições de ocupação devido à presença de sítios arqueológicos históricos e préhistóricos.

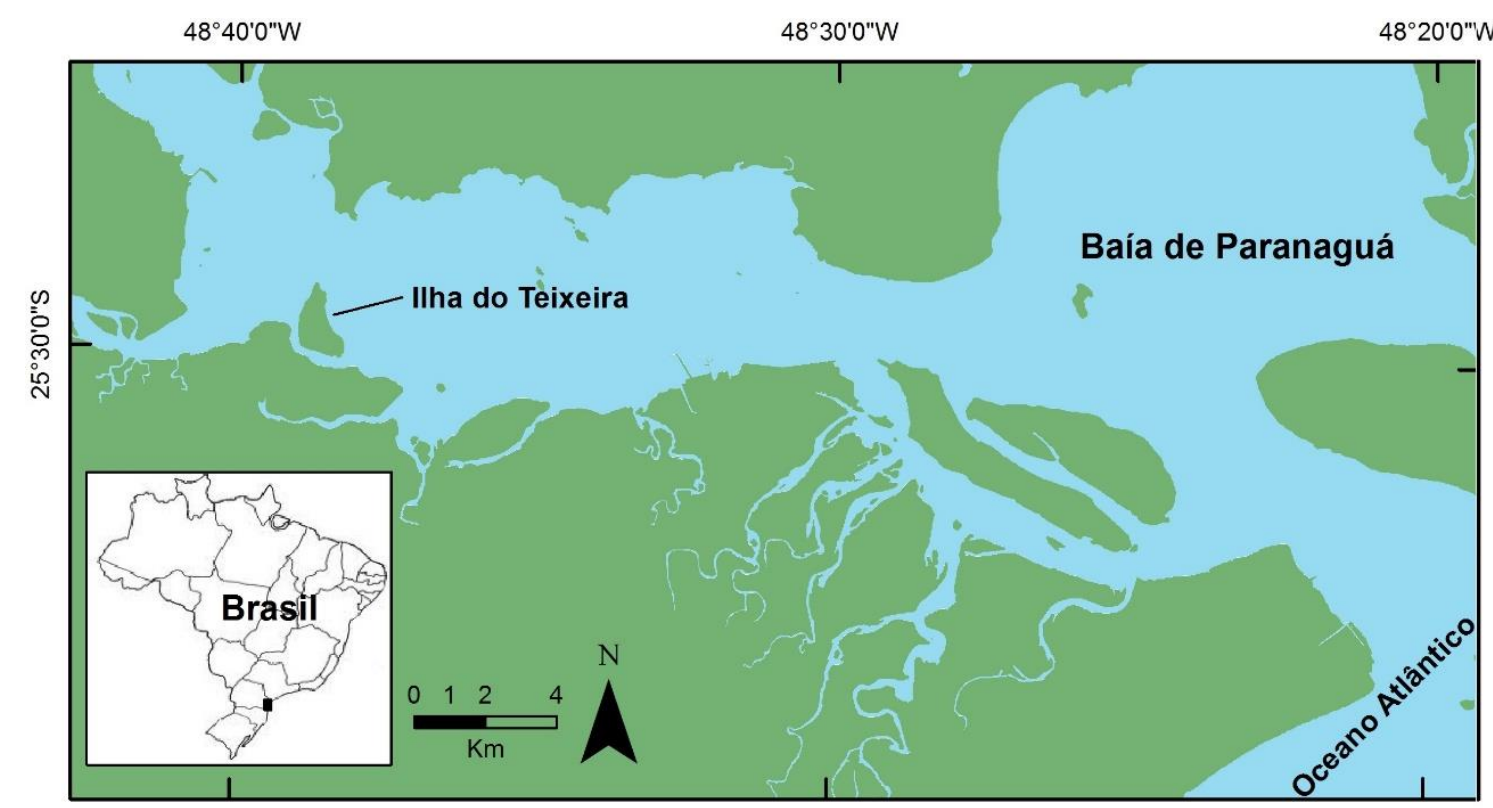

Figura 1. Localização da Ilha do Teixeira, na baía de Paranaguá, Estado do Paraná.

Os exemplares foram coletados manualmente em 14 de novembro de 2019, sendo encontrados através de prospecção visual em camadas internas do sambaqui da Ilha do Teixeira, as quais se apresentaram naturalmente erodidas. De acordo com Gernet \& Birckolz (2011), os sambaquis são formações artificiais constituídos, na sua maior parte, por conchas de moluscos que eram utilizadas tanto na construção destes monumentos quanto na alimentação dos povos pré-históricos (caçadores-pescadores-coletores) que habitaram o litoral paranaense.

O sambaqui em que foram encontrados os exemplares de Macrodontes paulistus está inserido no domínio da Floresta Ombrófila Densa, que apresenta diferentes fisionomias de acordo com as variações topográficas, climáticas e pedológicas, sendo então classificada como subformação de Floresta Ombrófila Densa de Terras Baixas (IBGE 1992). Tal subformação é caracterizada pela presença de numerosas epífitas, lianas e palmáceas, podendo apresentar variações discretas na composição e estrutura em virtude das condições de drenagem e 
fertilidade dos seus solos, basicamente em função de deposições aluviais, sendo o fator condicionante essencialmente edáfico (IPARDES 1995).

Três exemplares adultos de Macrodontes paulistus (Figura 2; Tabela 1) foram coletados, todos apresentando desgaste característico de conchas encontradas em sambaquis. As conchas apresentaram coloração esbranquiçada pela ação do tempo, não havendo a presença de perióstraco. Um dos exemplares estava com o perístoma fraturado. As conchas não mostravam sinais de utilização como objetos culturais (Gernet et al. 2019). Os exemplares foram depositados na Coleção Malacológica do Laboratório de Ecologia Aplicada e Bioinvasões da Universidade Federal do Paraná, Pontal do Paraná, Brasil (LEBIO 576).

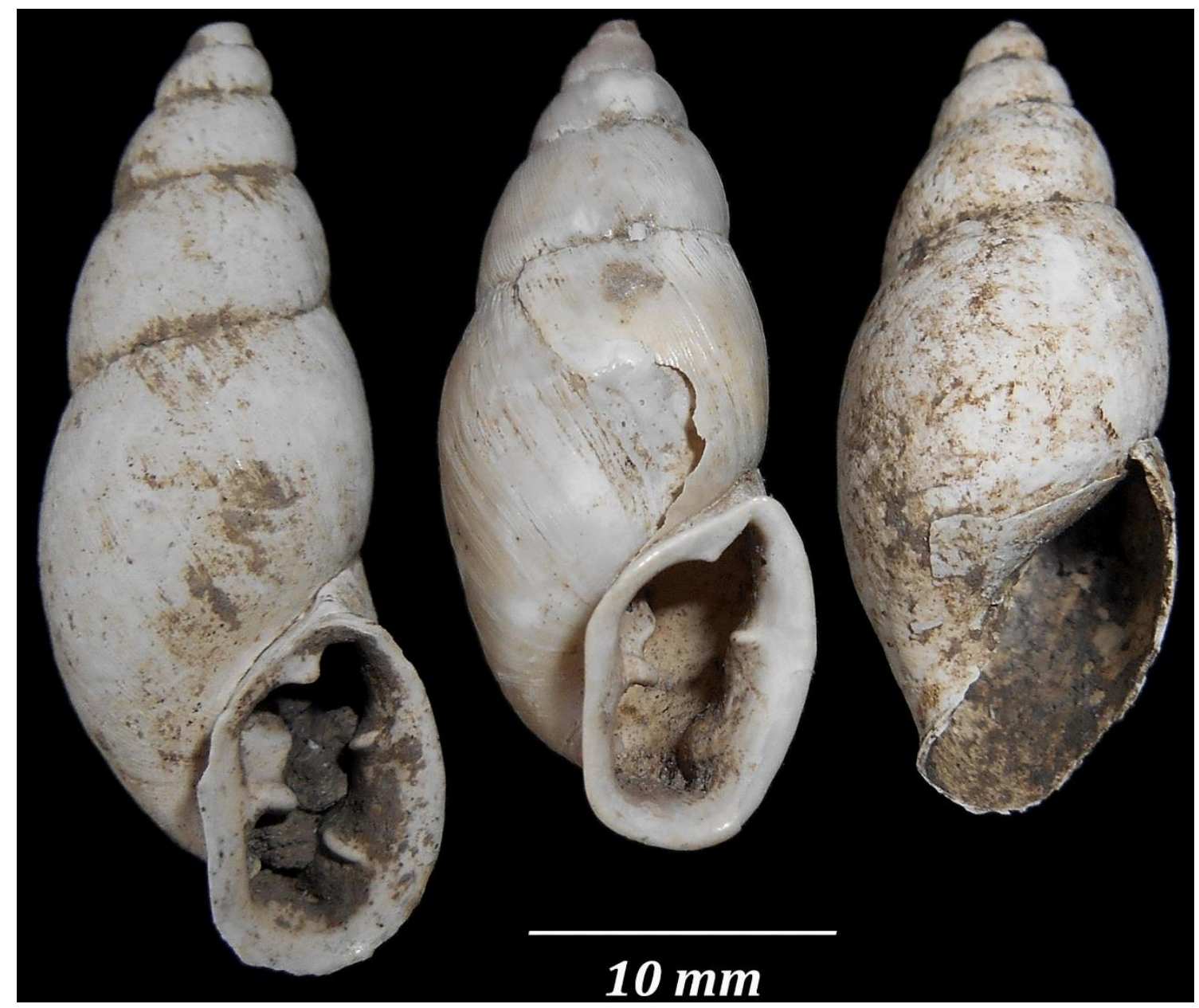

Figura 2. Conchas de Macrodontes paulistus (LEBIO 576) encontradas em sambaqui da Ilha do Teixeira, baía de Paranaguá, Estado do Paraná.

Como já demonstrado por outros autores (Gernet \& Birckolz 2011; Fontenelle et al. 2014; Macario et al. 2016, 2017), moluscos terrestres dos gêneros Megalobulimus K. Miller, 1878 e Thaumastus Albers, 1860 já foram encontrados em camadas de sambaquis nas regiões Sudeste e Sul do Brasil. Este registro aqui relatado para Macrodontes paulistus é o primeiro para o gênero em sambaquis.

Tabela 1. Medidas dos três exemplares de Macrodontes paulistus encontrados em sambaqui da Ilha do Teixeira, baía de Paranaguá, Estado do Paraná.

\begin{tabular}{cccc}
\hline Medidas & Exemplar 1 & Exemplar 2 & Exemplar 3 \\
\hline Comprimento & $31 \mathrm{~mm}$ & $26 \mathrm{~mm}$ & $25 \mathrm{~mm}$ \\
Largura maior & $10 \mathrm{~mm}$ & $09 \mathrm{~mm}$ & $10 \mathrm{~mm}$ \\
Comprimento da abertura & $11 \mathrm{~mm}$ & $10 \mathrm{~mm}$ & - \\
Largura da abertura & $07 \mathrm{~mm}$ & $06 \mathrm{~mm}$ & - \\
\hline
\end{tabular}




\section{Agradecimentos}

Agradecemos ao Prof. Dr. Luiz Ricardo Lopes de Simone (Universidade de São Paulo, Museu de Zoologia, Departamento de Zoologia) e aos avaliadores anônimos pela revisão crítica do manuscrito.

\section{Referências}

APPA, FEESC \& LABTRANS (2018) Plano de Desenvolvimento e Zoneamento do Porto de Paranaguá. Revisão - 005. Paranaguá: APPA. 368 p.

Fontenelle J.H., Cavallari D.C. \& Simone L.R.L. (2014) A new species of Megalobulimus (Gastropoda, Strophocheilidae) from Brazilian shell mounds. Strombus, 21(1-2): 30-37.

Gernet M.V. \& Birckolz C.J. (2011) Fauna malacológica em dois sambaquis do litoral do Estado do Paraná, Brasil. Biotemas, 24(3): 39-49. http://dx.doi.org/10.5007/2175-7925.2011v24n3p39

Gernet M.V., Gernet E.V.V.V. \& Santos E.V. (2019) Conchas de moluscos com vestígios de utilização por seres humanos encontradas no Sambaqui do Boguaçu, Guaratuba, Paraná. Pesquisa e Ensino em Ciências Exatas e da Natureza, 3(2): 142-146. http://dx.doi.org/10.29215/pecen.v3i2.1260

IBGE - Instituto Brasileiro de Geografia e Estatística (1992) Manual técnico da vegetação brasileira. Série Manuais Técnicos em Geociências (número 1). Rio de Janeiro: IBGE. 92 p.

IPARDES - Instituto Paranaense de Desenvolvimento Econômico e Social (1995) Diagnóstico Ambiental da APA de Guaraqueçaba. Curitiba: IPARDES. 166 p.

Maack R. (1969) Geografia Física do Estado do Paraná. Curitiba: BADEP/UFP/IBPT. 350 p.

Macario K.D., Alves E.Q., Carvalho C., Oliveira F.M., Ramsey C.B., Chivall D., Souza R., Simone L.R.L. \& Cavallari D.C. (2016) The use of the terrestrial snails of the genera Megalobulimus and Thaumastus as representatives of the atmospheric carbon reservoir. Scientific Reports, 6 : 27395. http://dx.doi.org/10.1038/srep27395

Macario K.D., Tenório M., Alves E., Oliveira F., Chanca I., Netto B., Carvalho C., Souza R., Aguilera O. \& Guimarães R. (2017) Terrestrial Mollusks as Chronological Records in Brazilian Shellmounds. Radiocarbon, 59(5): 1561-1577. https://doi.org/10.1017/RDC.2017.34

Oliveira M.P. \& Almeida M.N. (1999) Conchas dos caramujos terrestres do Brasil. Juiz de Fora: Editar. 57 p.

Pilsbry H.A. (1898) New species of Odontostomus from Brazil and Argentina. Proceedings of the Academy of Natural Sciences of Philadelphia, 50: 471-474.

Simone L.R.L. (2006) Land and Freshwater Molluscs of Brazil. São Paulo: EGB/Fapesp. 390 p.

Simone L.R.L. (2018) Physical defense strategies of South American land snails. Malacopedia, 1(2): 3-11. 